\title{
Histidine supplementation alleviates inflammation in the adipose tissue of high-fat diet-induced obese rats via the NF-кB- and PPAR $\gamma$-involved pathways
}

\author{
Xiaowei Sunt, Rennan Fengt, Yanchuan Li, Song Lin, Wei Zhang, Ying Li, Changhao Sun and \\ Songtao $\mathrm{Li}^{*}$ \\ Department of Nutrition and Food Hygiene, Public Health College, Harbin Medical University, 157 Baojian Road, \\ Nangang District, Harbin, Heilongjiang Province 150081, People's Republic of China
}

(Submitted 17 December 2013 - Final revision received 24 February 2014 - Accepted 18 March 2014 - First published online 15 May 2014)

\section{Abstract}

Obesity is considered to be accompanied by a chronic low-grade inflammatory state that contributes to the occurrence of many chronic diseases. Our previous study has demonstrated that histidine supplementation significantly ameliorates inflammation and oxidative stress in obese women. However, the in vivo potential mechanisms are not known. The present study was conducted to investigate the mechanisms underlying the effects of histidine on inflammation in a high-fat diet (HFD)-induced female obese rat model. An obese model was established in female Sprague-Dawley rats by HFD feeding for 8 weeks and followed by histidine supplementation for another 4 weeks. The results revealed that HFD-increased body weight and HFD-lowered serum histidine concentrations were significantly reversed by histidine supplementation $(P<0.05)$. In addition, the serum concentrations of TNF- $\alpha$, IL-6, C-reactive protein $(\mathrm{CRP})$ and malondialdehyde were significantly reduced and those of superoxide dismutase (SOD) were significantly increased by histidine supplementation when compared with those in obese rats $(P<0 \cdot 05)$. Correspondingly, the mRNA expressions of TNF- $\alpha$, IL- 6 and $C R P$ in the adipose tissue were significantly down-regulated and that of CUZnSOD was significantly up-regulated by histidine supplementation $(P<0 \cdot 05)$. Histidine supplementation significantly reduced the HFD-induced translocation of NF- $\kappa \mathrm{B}$ p65 into the nucleus $(P=0.032)$ by reducing the phosphorylation of the inhibitor of $\kappa \mathrm{B} \alpha$ in the adipose tissue. The results also revealed that the expression of adiponectin was markedly increased both in the serum and in the adipose tissue after histidine supplementation, accompanied by the activation of PPARy $(P=0.021)$. These findings indicate that histidine is an effective candidate for ameliorating inflammation and oxidative stress in obese individuals via the NF-кB- and PPAR $\gamma$-involved pathways.

\section{Key words: Histidine: High-fat diets: Obesity: Inflammation}

Obesity is a pro-inflammatory state characterised by an increased production of different cytokines by the adipose tissue $^{(1,2)}$. It has been reported that obesity-associated inflammation and oxidative stress are the key and early contributing factors for the occurrence of many chronic diseases, including type 2 diabetes, hypertension and $\mathrm{CVD}^{(1,3,4)}$. Therefore, the suppression of low-grade chronic pro-inflammation and oxidative stress is an effective strategy for reducing the risk of these diseases

Histidine is an important amino acid for humans. It has been reported that chronic kidney disease patients have a low plasma concentration of histidine, which is associated with protein-energy wasting, inflammation and oxidative stress ${ }^{(5)}$.
In a previous study, we had found that serum histidine concentrations in obese women were lower than those in non-obese women and had negative relationships with inflammation and oxidative stress ${ }^{(6)}$. The recovery of serum histidine concentrations to normal levels through histidine supplementation could significantly attenuate inflammation and oxidative stress in obese women ${ }^{(7)}$. In addition, plasma histidine concentrations have been found to be lower in diabetic $\mathrm{BALB} / \mathrm{cA}$ mice than in non-diabetic control mice, and histidine supplementation has been found to markedly reduce the concentrations of IL- 6, TNF- $\alpha$ and C-reactive protein $(\mathrm{CRP})^{(8)}$. Histidine has been reported to inhibit the TNF- $\alpha$-induced $I L-8$ expression at the transcriptional level in intestinal

\footnotetext{
Abbreviations: CRP, C-reactive protein; HFD, high-fat diet; H-His, high histidine dose group; HH DR, diet-restricted group for high histidine dose; IкB $\alpha$, inhibitor of $\kappa \mathrm{B} \alpha$; OM, obese model group; OM DR, diet-restricted group for obese model; SOD, superoxide dismutase
}

*Corresponding author: Dr S. Li, fax +86 45187502885 , email lisongtaoklds@126.com

† These authors contributed equally to the present study. 
epithelial cells ${ }^{(9)}$. However, the potential in vivo mechanisms involved in the anti-inflammatory and anti-oxidative stress functions of histidine in the obese state are still unclear.

The transcription factor NF- $\mathrm{BB}$ is a central regulator of various cellular genes involved in immune and inflammatory responses $^{(10,11)}$. Under basal conditions, NF- $\kappa \mathrm{B}$ is an inactive cytoplasmic heterotrimer consisting of p50, p65 and inhibitor of $\kappa \mathrm{B} \alpha \quad(\mathrm{I} \kappa \mathrm{B} \alpha)$ subunits. In response to stimulation by factors such as lipopolysaccharides and TNF- $\alpha$, IкB $\alpha$ undergoes phosphorylation and an ubiquitination-dependent degradation by a proteasome complex, which leads to the p65 subunit being transferred into the nucleus and stimulating the transcription of its target genes, such as $I L-6, T N F-\alpha$ and $C R P^{(10)}$. The reported studies have revealed that the NF-кB signalling pathway could be a therapeutic target in chronic inflammation, evidenced by the results that the inhibition of this pathway could attenuate inflammatory responses ${ }^{(12,13)}$.

Adiponectin, one of the most abundant gene transcripts of the adipose tissue, is an important link among obesity, inflammation and chronic diseases ${ }^{(14,15)}$. It has been reported that an increase in adiponectin concentrations could attenuate inflammation in obese individuals ${ }^{(16)}$. In a previous study, we had found that histidine supplementation could increase the serum concentrations of adiponectin in obese women ${ }^{(7)}$. Therefore, we sought to study whether histidine could attenuate inflammation by increasing the serum concentrations of adiponectin. The expression of adiponectin has been found to be significantly enhanced by PPAR $\gamma$ at the transcriptional level $^{(17)}$. PPAR $\gamma$ is a ligand-activated transcription factor that is most widely expressed in the adipose tissue. Importantly, PPAR $\gamma$ is a central regulator of adipocyte differentiation and controls many adipocyte genes by binding to specific PPAR response elements in the promoters of these genes ${ }^{(18)}$

Therefore, in the present study, we focused on the NF-кBand PPAR $\gamma$-involved pathways to investigate the mechanisms underlying the effects of histidine on inflammation and oxidative stress in a high-fat diet (HFD)-induced female obese rat model.

\section{Experimental methods}

\section{Ethics statement}

All protocols used in the present study were approved by the Medical Ethics Committee of Harbin Medical University (Harbin, China) and were implemented in accordance with the National Institutes of Health regulations for the care and use of animals in research.

\section{Animals and obese model}

A total of fifty adult female Sprague-Dawley rats with body weight ranging from 160 to $200 \mathrm{~g}$ were purchased from Shanghai SLACK Laboratory Animal. The rats were allowed to acclimatise for 1 week before conducting the experiment. Among these rats, eight were randomly selected and fed the AIN-93M diet for 12 weeks until the end of the experiment ${ }^{(19)}$. The remaining forty-two rats were fed a HFD for 8 weeks to establish an obese model (OM group). The HFD was based on the AIN-93M diet with lard and maize starch contents being adjusted to 180 and $286.5 \mathrm{~g} / \mathrm{kg}$, respectively. To ensure that both diets had the same amount of amino acids, especially histidine, the average food intake per $\mathrm{d}$ of rats fed the AIN-93M diet was restricted to the same amount of food intake of the OM group, and the eight rats were designated as the diet-restricted group for obese model (OM DR group). The rats were housed individually in a temperaturecontrolled room under a $12 \mathrm{~h}$ light $-12 \mathrm{~h}$ dark cycle and given free access to water.

\section{Histidine supplementation}

A total of ten HFD-fed rats with body weight less than the mean body weight plus 1-fold of the standard deviation of the OM DR group were considered to be obesity resistant and excluded from the study. The remaining obese rats ( $n 32)$ were randomly assigned to four groups: (1) OM group (no additional histidine included); (2) low-histidine dose group (histidine $0.375 \mathrm{~g} /$ kg.body weight); (3) high-histidine dose (H-His) group (histidine $1.875 \mathrm{~g} / \mathrm{kg} \cdot$ body weight); (4) diet-restricted group for high histidine dose (HH DR) group (no additional histidine included and with food intake the same as that of the H-His group). Histidine (purity $\geq 95 \%$ ) was purchased from Yuan Cheng Gong Chuang. The four groups were fed the same HFD mentioned above until the end of the experiment. Suspensions containing different doses of histidine in an aqueous solution of carboxymethyl cellulose were orally administered to rats at a dose of $1 \mathrm{ml} / 100 \mathrm{~g} \cdot$ body weight. These doses were chose on the basis of the protocols described in previous reports ${ }^{(20,21)}$. Treatment was carried out for four consecutive weeks (Fig. 1). Food intake was recorded daily and body weight was measured weekly.

\section{Sample collection}

Tail blood samples were collected from each rat at the beginning and at the end of week 8 . At week 12, the rats were killed after an overnight fast and pentobarbital anaesthesia. Blood samples were obtained from the abdominal aorta and centrifuged ( $3000 \mathrm{rpm}$ for $15 \mathrm{~min}$ ). Livers, kidneys and white adipose tissues (parametrium, perirenal fat and omental fat pads) were removed, weighed, quick-frozen in liquid $\mathrm{N}_{2}$ and stored at $-80^{\circ} \mathrm{C}$ for further analysis.

\section{Serum analysis}

The serum concentrations of histidine were measured at weeks 0,8 and 12 according to the method reported previously $^{(6)}$. The serum concentrations of TNF- $\alpha$, IL-6, CRP and adiponectin were determined by ELISA using commercial kits (TNF- $\alpha$ and IL-6, R\&D Systems Europe; CRP, Biocheck, Inc.; and adiponectin, AdipoGen) according to the manufacturers' protocols. The serum concentrations of superoxide dismutase (SOD) and malondialdehyde were measured with enzymatic methods using commercial kits (Jiancheng Technology). 


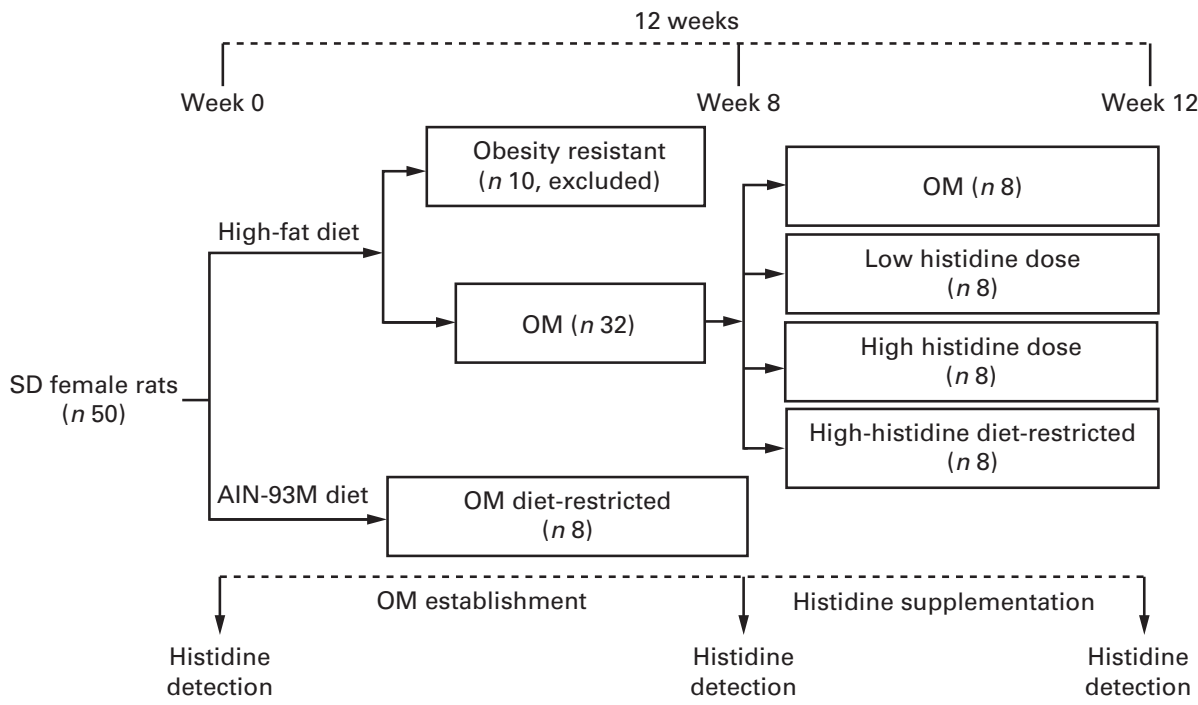

Fig. 1. Flowchart depicting obese model (OM) establishment and histidine supplementation protocol. SD, Sprague-Dawley.

\section{RNA isolation and real-time $P C R$}

The mRNA levels of $T N F-\alpha, I L-6, C R P$, adiponectin and CuZn$S O D$ in white adipose tissue were determined by real-time PCR. Total mRNA was extracted from the white adipose tissue using the TRIzol reagent (Invitrogen). Real-time PCR was carried out using the SYBR Green PCR Master Mix and the 7500 FAST Real-time PCR System (Applied Biosystems). Relative quantification of mRNA expression was performed using the $\Delta \Delta C_{\mathrm{t}}\left(2^{-\Delta \Delta C_{\mathrm{t}}}\right) \operatorname{method}^{(22)}$. Primer sequences used in the PCR are listed in Table 1 . All reactions were carried out at least in triplicate.

\section{Western blot analysis}

Nuclear protein extracts and total protein extracts of the white adipose tissue were prepared using protein extraction kits (Biotype Institute of Biotechnology) according to the manufacturers' protocol. Western blot analysis was carried out as described previously to measure the concentrations of proteins involved in the NF- $\mathrm{B}$ and PPAR $\gamma$ pathways ${ }^{(23)}$. Antibodies used for protein detection were bought from the

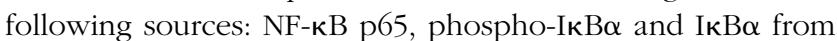
Cell Signaling Technology; PPAR $\gamma$ and Histone $\mathrm{H} 3$ from Abcam; $\beta$-actin from Santa Cruz Biotechnology, Inc.; secondary antibody from Promega Corporation. Data are presented as the relative intensity of the protein bands. Experiments were replicated at least three times, and representative blots are shown in the figures.

\section{Statistical analysis}

Data are presented as means with their standard errors. Data were analysed using one-way ANOVA followed by post hoc test, with $P<0.05$ considered to be significant. All $P$ values were two-sided. Statistical analysis was carried out using the SPSS software (version 16; Beijing Stats Data Mining).

\section{Results}

Histidine reduces high-fat diet-induced body weight increase and food intake

An obese rat model was successfully established after 8 weeks of HFD feeding, evidenced by the obvious increase in body weight in the OM group when compared with that in the OM DR group ( $P<0 \cdot 001$; Table 2$)$. After histidine supplementation for another 4 weeks, HFD-induced body weight increase was markedly reduced by high-dose histidine supplementation compared with that in OM group ( $P=0.042$; Table 2$)$. The food intake of the H-His group was significantly lower than that of the OM group from week $10(P=0.026)$ after histidine supplementation (Fig. 2). However, no significant differences in body weight and food intake were observed between the $\mathrm{H}-\mathrm{His}$ and HH DR groups.

\section{Histidine supplementation reverses high-fat diet-lowered serum histidine concentrations in obese rats}

There was no difference in serum histidine concentrations between the OM and OM DR groups at the start of the

Table 1. Primer sequences for $T N F-\alpha, I L-6$, C-reactive protein (CRP), copper-zinc superoxide dismutase $(C u Z n S O D)$, adiponectin and $\beta$-actin used in the PCR

\begin{tabular}{ll}
\hline Genes & Primer sequences \\
\hline$T N F-\alpha$ & Forward: $5^{\prime}$-TGTCTCAGCCTCTTCTCATT-3' \\
& Reverse: 5'-AGATGATCTGAGTGTGAGGG-3' \\
IL-6 & Forward: 5'-GCCACTGCCTTCCCTACTTCA-3' \\
CRP & Reverse: 5'-GACAGTGCATCATCGCTGTTCA-3' \\
& Forward: 5'-GTAGGTGGGCCTGAAATACTGTTC-3' \\
CuZnSOD & Reverse: 5'-AAGCCAAAGCTCTACAATTCCTGT-3' \\
Adiponectin & Forward: 5'-GTTCCGAGGCCGCGCGT-3' \\
& Reverse: 5'-GTCCCCATATTGATGGAC-3' \\
3-Actin & Forward: 5'-GGCCGTTCTCTTCACCTACG-3' \\
& Reverse: 5'-GGCTCCATGCTCCTCCATCT-3' \\
& Forward: 5'-AGGGAAATCGTGCGTGAC-3' \\
\hline
\end{tabular}


Table 2. Serum histidine concentration and body weight before and after histidine supplementation in rats

(Mean values with their standard errors)

\begin{tabular}{|c|c|c|c|c|c|c|}
\hline \multirow[b]{2}{*}{ Weeks } & \multirow[b]{2}{*}{ Groups } & \multirow[b]{2}{*}{$n$} & \multicolumn{2}{|c|}{$\begin{array}{c}\text { Histidine } \\
\text { concentration } \\
(\mu \mathrm{mol} / \mathrm{l})\end{array}$} & \multicolumn{2}{|c|}{$\begin{array}{c}\text { Body } \\
\text { weight (g) }\end{array}$} \\
\hline & & & Mean & SEM & Mean & SEM \\
\hline \multirow[t]{2}{*}{0} & OM DR & 8 & $97 \cdot 70$ & 3.37 & 184.9 & $4 \cdot 2$ \\
\hline & OM & 32 & 98.96 & 1.94 & $186 \cdot 1$ & $2 \cdot 3$ \\
\hline \multirow[t]{2}{*}{8} & OM DR & 8 & $83 \cdot 10^{*}$ & 3.67 & $270 \cdot 0^{\star \star}$ & 6.5 \\
\hline & $\mathrm{OM}$ & 32 & 73.07 & $2 \cdot 11$ & 306.5 & 4.8 \\
\hline \multirow[t]{5}{*}{12} & OM DR & 8 & $68.59^{\star \star}$ & 2.59 & $299 \cdot 5^{\star *}$ & $5 \cdot 6$ \\
\hline & $\mathrm{OM}$ & 8 & $55 \cdot 78$ & 1.97 & $347 \cdot 3$ & $5 \cdot 3$ \\
\hline & L-His & 8 & 59.97 & 2.37 & 336.4 & $5 \cdot 1$ \\
\hline & $\mathrm{H}-\mathrm{His}$ & 8 & $67.97^{\star \star}$ & 2.49 & $325 \cdot 3^{*}$ & 4.6 \\
\hline & HH DR & 8 & 57.47 & $2 \cdot 32$ & $324 \cdot 9^{\star}$ & 4.7 \\
\hline
\end{tabular}

OM, obese model group; OM DR, diet-restricted group for obese model; L-His, low-histidine dose group; $\mathrm{H}$-His, high-histidine dose group; HH DR, diet-restricted group for high histidine dose.

Mean values were significantly different from those of the OM group: ${ }^{*} P<0.05$; ${ }^{\star *} P<0.01$ (ANOVA).

experiment (Table 2). After HFD feeding for 8 weeks, serum histidine concentrations were decreased by $12 \%$ in the OM group, compared with those in the OM DR group $(P=0.036$; Table 2). Compared with those in HH DR group, the HFDlowered serum concentrations of histidine were significantly reversed by histidine supplementation in the $\mathrm{H}$-His group $(P=0 \cdot 032$; Table 2$)$.

\section{Histidine alleviates high-fat diet-induced adverse changes} in serum inflammatory and oxidative biomarkers

As shown in Fig. 3, compared with those in the OM DR group, the serum concentrations of inflammatory factors, including TNF- $\alpha$, IL- 6 and CRP, were significantly elevated by HFD feeding in the OM group $(P=0.007, P=0.004$ and $P=0.005$, respectively). The serum concentrations of adiponectin were reduced by HFD feeding $(P=0 \cdot 002)$. After histidine supplementation, inflammation was not significantly attenuated in the low-histidine dose group $(P>0 \cdot 05)$. The HFD-induced adverse changes in inflammatory factors mentioned above were significantly alleviated in the $\mathrm{H}$-His group than in the HH DR group $(P=0.020$, Fig. 3(A); $P=0.035$, Fig. 3(B); $P=0.031$, Fig. $3(\mathrm{C})$; and $P=0.028$, Fig. 3(D), respectively). The results also revealed that histidine supplementation significantly improved HFD-lowered SOD concentrations $(P=0.017)$ and HFD-increased malondialdehyde concentrations $(P=0.039)$ in the serum when compared with those in the HH DR group (Fig. 3(E) and (F)).

\section{Histidine ameliorates the MRNA expressions of inflammatory and oxidative biomarkers in the adipose tissue}

The mRNA expressions of TNF- $\alpha, I L-6$ and $C R P$ in the adipose tissue were markedly increased by HFD feeding $(P<0 \cdot 001)$. Histidine supplementation significantly alleviated HFD-increased
$T N F-\alpha, I L-6$ and $C R P$ expressions in the adipose tissue in the $\mathrm{H}-\mathrm{His}$ group than in the HH DR group $(P=0 \cdot 011$, Fig. 4(A); $P=0.043$, Fig. 4(B); and $P=0.037$, Fig. 4(C), respectively). In addition, HFD-lowered adiponectin and CUZnSOD expressions in the adipose tissue were also reversed by high-dose histidine supplementation at the mRNA level compared with those in the HH DR group $(P=0.019$, Fig. 4(D), and $P=0 \cdot 013$, Fig. 4(E)).

\section{$N F-\kappa B$ pathway is involved in the anti-inflammatory role of histidine}

The HFD stimulated strong transference of p65 into the nucleus in the adipose tissue compared with that in the OM DR group $(P<0 \cdot 001)$. High-dose histidine supplementation significantly reduced the nuclear p65 content, accompanied by a decreased ratio of $\mathrm{p} 65$ protein content in the nucleus to that in the cytoplasm in the adipose tissue $(P=0.032$; Fig. 5(A) and (B)). Histidine supplementation also blocked the phosphorylation of $\mathrm{I} \kappa \mathrm{B} \alpha$ in the adipose tissue $(P=0.024$; Fig. 5(C) and (D)). These results indicate that the histidineinhibited NF- $\mathrm{B}$ pathway contributes to its anti-inflammatory role in the adipose tissue.

\section{Histidine induces PPAR $\gamma$ expression in the adipose tissue}

After 8 weeks of HFD feeding, the protein expression of PPAR $\gamma$ in the adipose tissue of the OM group was decreased compared with that in the adipose tissue of the OM DR group $(P<0 \cdot 001)$. High-dose histidine supplementation significantly improved the HFD-down-regulated expression of PPAR $\gamma$ at the protein level $(P=0.021)$, suggesting that the PPAR $\gamma$ pathway is probably involved in the upregulation effects of histidine on adiponectin expression (Fig. 6(A) and (B)).

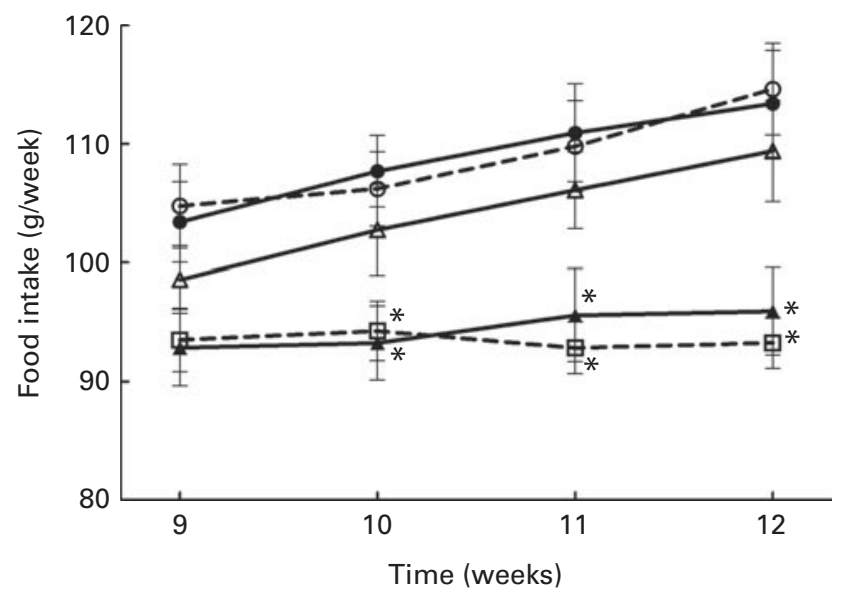

Fig. 2. Comparison of weekly food intake of each group after histidine supplementation. OM (-)), obese model group; OM DR (- $\ominus$-), diet-restricted group for obese model; L-His $\left(\triangle_{-}\right)$, low-histidine dose group; $\mathrm{H}$-His $\left(-\mathbf{A}_{-}\right)$, high-histidine dose group; HH DR (-曰), diet-restricted group for high histidine dose. Values are means, with their standard errors represented by vertical bars. ${ }^{*}$ Mean values were significantly different from those of the OM group $(P<0.05$; ANOVA). 
(A)

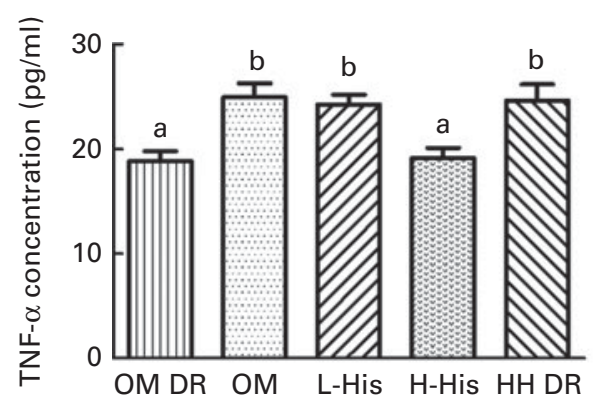

(B)

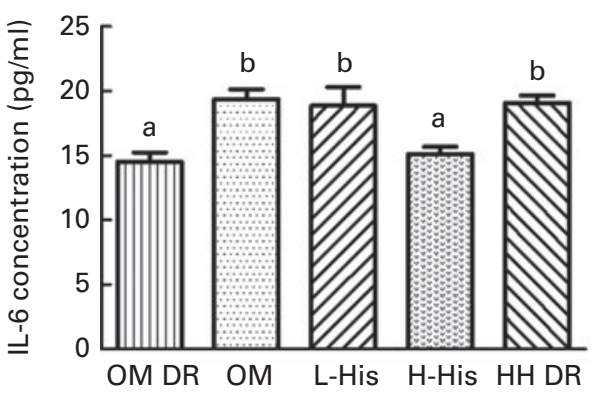

(C)

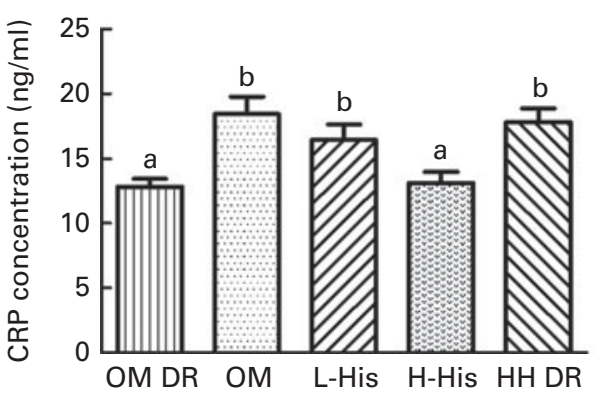

(D)

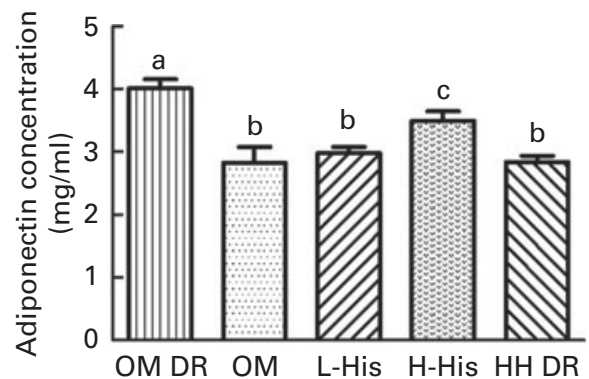

(E)

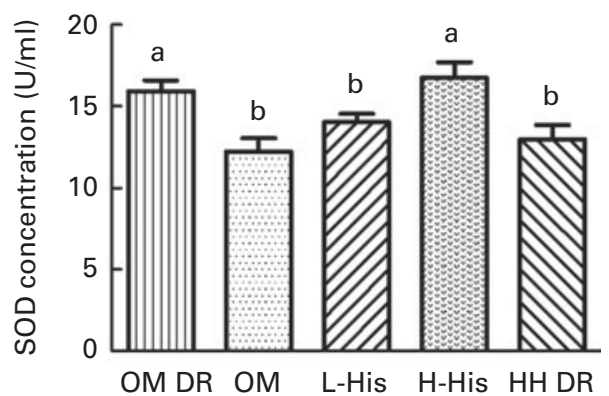

(F)

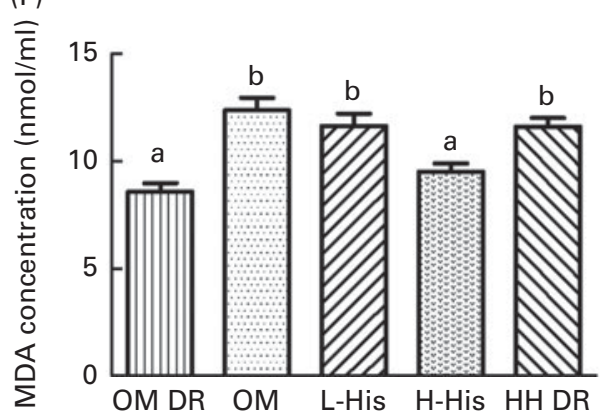

Fig. 3. Effects of histidine supplementation on serum inflammatory and oxidative biomarkers. The serum concentrations of (A) TNF- $\alpha$, (B) IL-6, (C) C-reactive protein (CRP) and (D) adiponectin were determined using commercial ELISA according to the manufacturers' protocols and those of (E) superoxide dismutase $(\mathrm{SOD})$ and $(\mathrm{F})$ malondialdehyde (MDA) were measured with enzymatic methods using commercial kits. Values are means, with their standard errors represented by vertical bars. ${ }^{\mathrm{a}, \mathrm{b}, \mathrm{c}}$ Mean values with unlike letters were significantly different $(P<0.05$; ANOVA). OM, obese model group; OM DR, diet-restricted group for obese model; L-His, low-histidine dose group; H-His, high-histidine dose group; HH DR, diet-restricted group for high histidine dose.

\section{Discussion}

The present study demonstrates for the first time that histidine supplementation ameliorates HFD-induced inflammation in the adipose tissue of obese rats. The results of the present study revealed that histidine exerted its beneficial effects on obesity-associated inflammation by inactivating the NF-кB signalling pathway. The results also revealed that histidine reversed HFD-lowered adiponectin expression via a PPAR $\gamma$-involved pathway, which might contribute to its role in anti-inflammation.

Histidine is generally considered to be a dietary essential amino acid for human infants and an important amino acid for adults. It has been reported that chronic kidney disease patients and obese and type 2 diabetic youth have low plasma concentrations of histidine ${ }^{(5,24)}$. In our previous study, we had reported serum histidine concentrations were lower in obese women ${ }^{(6)}$. A similar result was obtained in the present study, i.e. serum concentrations of histidine in the HFD-induced obese rats were lower than those in the normal diet-fed non-obese rats, suggesting that the serum amino acid metabolism is correlated with the occurrence of obesity. In addition, we also observed that food intake and weight gain were lower in the H-His group than in the OM group, which could probably be explained by the suppression of appetite by histidine through its conversion into neuronal histamine in the hypothalamus $^{(25,26)}$. To eliminate the food intake-induced disturbance, we established a HH DR group for further excluding the effects of the reduced body weight on the alleviation of inflammation and, thereby, focused on the inhibitory effects of histidine on inflammation and oxidative stress.

TNF- $\alpha$, IL- 6 and CRP are pro-inflammatory cytokines, and the elevation of their concentrations has been confirmed in obese subjects ${ }^{(1,2)}$. Previous studies have suggested that TNF- $\alpha$ and IL-6 are involved in obesity-related insulin resistance and atherosclerosis ${ }^{(27,28)}$. CRP is the most extensively 
(A)

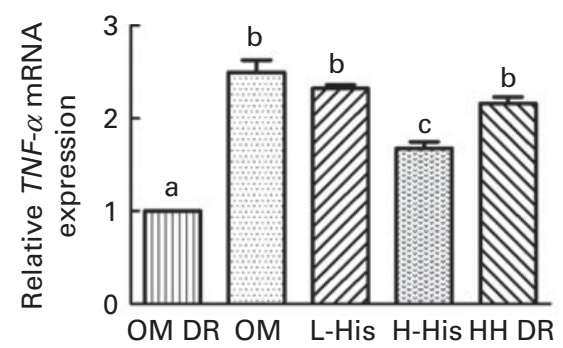

(B)

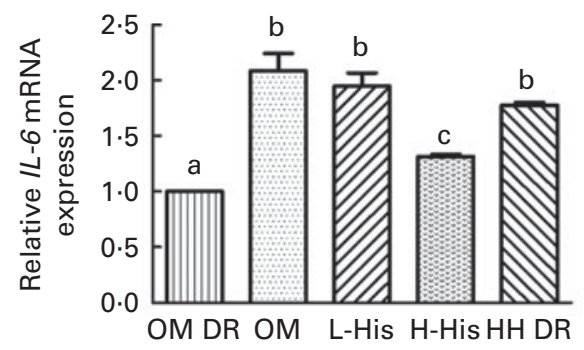

(C)

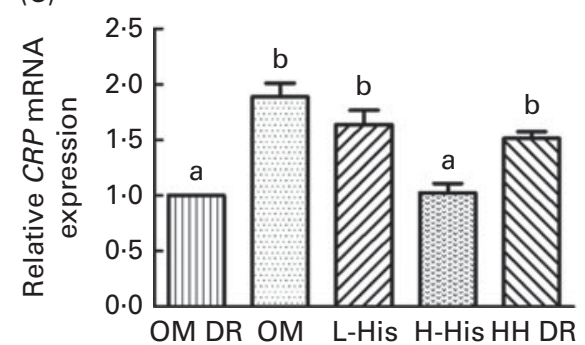

(D)

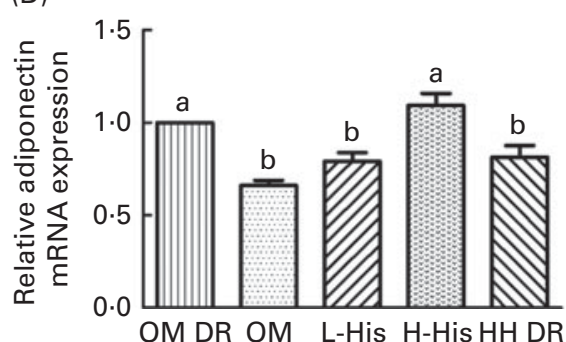

(E)

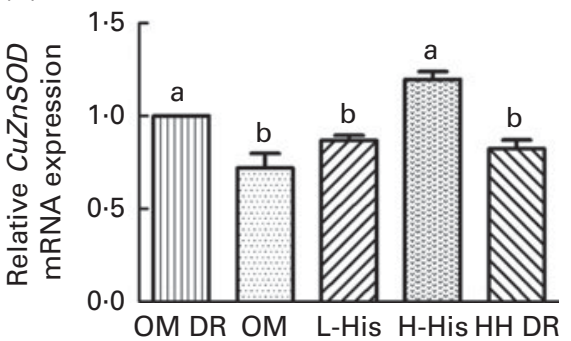

Fig. 4. Effects of histidine supplementation on the mRNA expressions of inflammatory and oxidative biomarkers in the adipose tissue. Adipose tissue mRNA was extracted and the mRNA expressions of (A) TNF- $\alpha$, (B) IL-6, (C) C-reactive protein (CRP), (D) adiponectin and (E) copper-zinc superoxide dismutase (CuZnSOD) were measured by quantitative real-time PCR. Expression was normalised to that of $\beta$-actin. Values are means, with their standard errors represented by vertical bars. ${ }^{\mathrm{a}, \mathrm{b}, \mathrm{c}}$ Mean values with unlike letters were significantly different $(P<0.05$; ANOVA). OM, obese model group; OM DR, diet-restricted group for obese model group; L-His, low-histidine dose group; H-His, high-histidine dose group; HH DR, diet-restricted group for high histidine dose.

studied marker of systemic inflammation in humans, which is also related to the insulin resistance syndrome and endothelial dysfunction $^{(29)}$. In the present study, HFD induced an increase in the serum concentrations of TNF- $\alpha$, IL- 6 and CRP in the OM group but not in the OM DR group. Moreover, we found that high-dose histidine supplementation could decrease the serum concentrations of TNF- $\alpha$, IL- 6 and CRP in the H-His group but not in HH DR group, in spite of the same amount of food being consumed by both groups. These results were consistent with the results of our previous study in which histidine supplementation was found to significantly lower the serum concentrations of the inflammatory biomarkers TNF- $\alpha$ and IL-6 in obese women ${ }^{(7)}$. Correspondingly, a previous study has reported that plasma histidine concentration is significantly lower in chronic kidney disease patients with a history of CVD and inflammation, which is associated with inflammation, oxidative stress and mortality ${ }^{(5)}$. The results of an animal study revealed that diabetic BALB/CA mice had
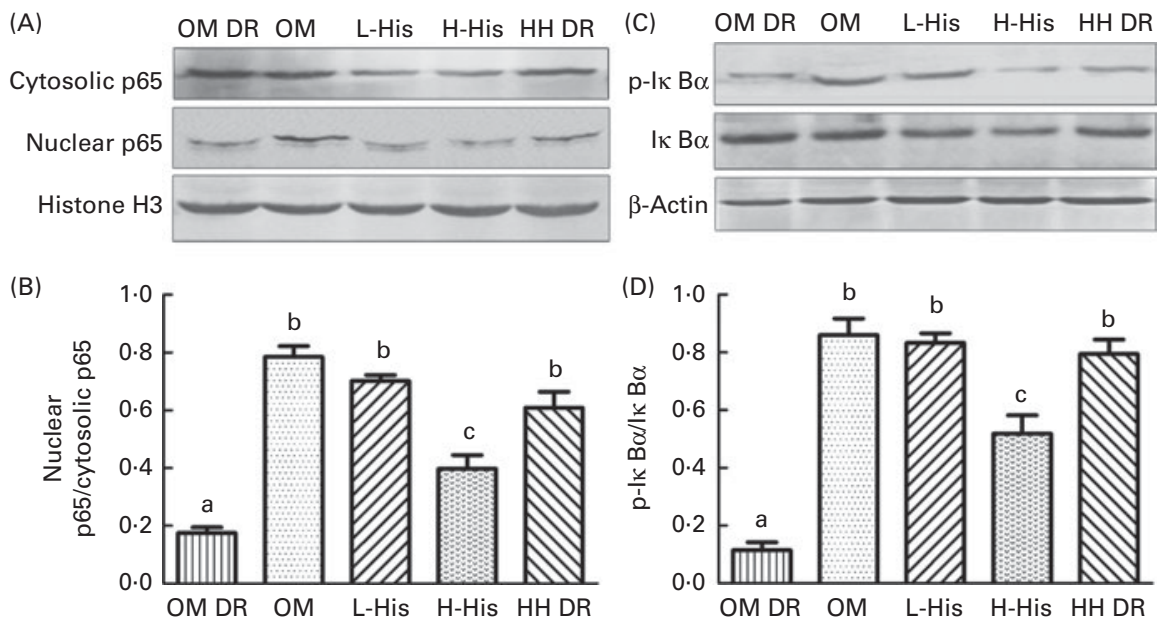

Fig. 5. Inhibitory effects of histidine supplementation on the NF-кB-involved pathway in the adipose tissue. Nuclear protein extracts and total cell protein of the white adipose tissue were prepared and measured by Western blot analysis. (A) Blot depicting the reduction of p65 transference into the nucleus by histidine. (B) Quantification of the protein expression of p65 in the nucleus and cytoplasm shown as the relative intensity of the protein bands. (C) Blot showing the inhibition of the phosphorylation (p) of the inhibitor of $\kappa B \alpha(I \kappa B \alpha)$ by histidine. (D) Quantification of the protein expression of $p$-I $\mathrm{B} \alpha$ shown as the relative intensity of the protein bands. Values are means, with their standard errors represented by vertical bars. ${ }^{\mathrm{a}, \mathrm{b}, \mathrm{c}}$ Mean values with unlike letters were significantly different $(P<0.05$; ANOVA). OM, obese model group; OM DR, diet-restricted group for obese model; L-His, low-histidine dose group; H-His, high-histidine dose group; HH DR, dietrestricted group for high histidine dose. 


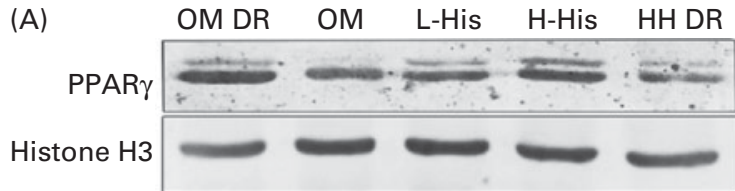

(B)

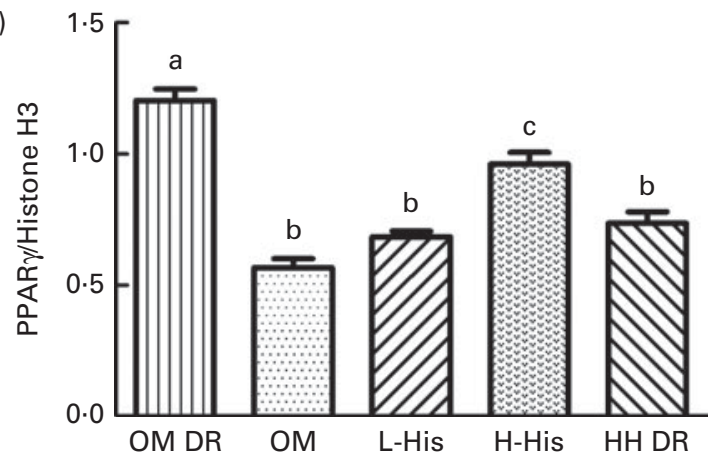

Fig. 6. Effects of histidine supplementation on the protein expression of PPAR $\gamma$ in the adipose tissue. Nuclear protein extracts of the white adipose tissue were prepared and measured by Western blot analysis. (A) Blot depicting the induction of PPAR $\gamma$ protein expression in adipose tissue by histidine. (B) Relative densities normalised to Histone $\mathrm{H} 3$ expression. Values are means, with their standard errors represented by vertical bars. ${ }^{a, b, c}$ Mean values with unlike letters were significantly different $(P<0.05$; ANOVA). OM, obese model group; OM DR, diet-restricted group for obese model; L-His, low-histidine dose group; H-His, high-histidine dose group; HH DR, dietrestricted group for high histidine dose.

lower plasma histidine concentrations than non-diabetic control mice and histidine supplementation could reduce the concentrations of IL- 6, TNF- $\alpha$ and CRP in diabetic animal models $^{(8)}$. Besides, an in vitro study has also reported that histidine has a potential to attenuate intestinal inflammation by inhibiting the $\mathrm{H}_{2} \mathrm{O}_{2}$ and TNF- $\alpha$-induced IL- 8 secretion at the transcriptional level in intestinal epithelial cells ${ }^{(9)}$

Adipose tissue is a crucial endocrine organ that secretes a wide variety of cytokines and has a central role in obesityassociated complications such as dyslipidaemia, insulin resistance and type 2 diabetes. Several studies have revealed that attenuation of inflammation could reduce the occurrence of inflammation-related diseases ${ }^{(30,31)}$. It has been reported that pre-intake of histidine and carnosine decreases the hepatic concentrations of IL- 6 , IL- 10 , and TNF- $\alpha$ during acetaminophen-induced liver injury in BALB/cA mice ${ }^{(20)}$. Our previous study has also demonstrated that histidine could inhibit the expressions of $I L-6$ and $T N F-\alpha$ in human preadipocytes $^{(7)}$. However, little is known about the anti-inflammatory effects of histidine in the adipose tissue. Therefore, we quantified the mRNA expressions of inflammatory biomarkers in the adipose tissue using real-time PCR. The results revealed that the mRNA expressions of $T N F-\alpha, I L-6$, and $C R P$ in the adipose tissue were down-regulated by high-dose histidine supplementation, which indicates that histidine could alleviate HFD-induced inflammation in the adipose tissue. A number of recent reports have demonstrated the key role of the NF- $\mathrm{B}$ signalling pathway in the development of inflammationassociated metabolic disorders in the liver and adipose tissue $^{(32,33)}$. The transcription factor NF- $\kappa \mathrm{B}$ is a primary regulator of inflammatory responses. It has been reported that histidine could be a novel therapeutic agent for Crohn's disease by inhibiting NF- $\mathrm{B}$ activation and down-regulating inflammatory cytokine production in macrophages ${ }^{(34)}$. Besides, in the previous study, we had observed that histidine could inhibit the translocation of NF- $\mathrm{KB}$ into the nucleus in human preadipocytes ${ }^{(7)}$. So, we quantified the expression of proteins involved in the NF-кB pathway in the adipose tissue by Western blot analysis, and the results revealed that histidine significantly inhibited NF- $\mathrm{B}$ p 65 translocation and I $\mathrm{B} \alpha$ degradation, which suggests that NF- $\mathrm{B}$ might be responsible for the anti-inflammatory effects of histidine.

Adiponectin is an anti-inflammatory factor secreted by adipocytes $^{(16)}$. In contrast to most adipocyte hormones, the concentrations of circulating adiponectin are decreased in obesity and increased in response to weight loss ${ }^{(35)}$. We had found that histidine supplementation could increase the serum concentrations of adiponectin in obese women ${ }^{(7)}$. In the present study, we quantified the concentration of adiponectin in the serum and its mRNA expression in the adipose tissue. The results revealed that the expression of adiponectin was significantly increased by histidine supplementation. It is well accepted that the expression of adiponectin is markedly stimulated by the activation of PPAR $\gamma$ in adipocytes ${ }^{(17)}$. So, we quantified the protein expression of PPAR $\gamma$ in the adipose tissue, and the results revealed that the expression of PPAR $\gamma$ was up-regulated by histidine supplementation, which might at least partially explain the results obtained for the effects of histidine supplementation on adiponectin. Moreover, PPAR $\gamma$ and NF- $\mathrm{BB}$ interact in several ways to oppose their respective activities. PPAR $\gamma$ can form a transcriptionally inhibitory complex with $\mathrm{NF}-\kappa \mathrm{B}$ in mouse macrophages ${ }^{(36)}$ Another study has revealed that the activation of PPAR $\gamma$ suppresses cytokine-induced NF- $\kappa \mathrm{B}$ transcriptional activity and target gene expression in skeletal muscle cells ${ }^{(37)}$. NF- $\kappa \mathrm{B}$ also inhibits the binding of PPAR to genomic response elements, thereby reducing the transcriptional activity of PPAR and the expression of PPAR-related transcripts ${ }^{(38)}$.

In addition, histidine has been reported to protect human LDL against oxidation and glycation and to have beneficial effects on liver in rats with acetaminophen-induced liver injury through its actions against oxidative stress ${ }^{(8,20)}$. Histidine can also restrict the accumulation of free radicals and delay the activation of extracellular signal-regulated kinase and c-jun N-terminal kinase in neuronal cells ${ }^{(39)}$. Previous studies have suggested that the anti-oxidative effect of histidine is based on its free radical-scavenging and divalent metal ion-chelating abilities ${ }^{(40,41)}$. In the present study, we quantified two oxidative biomarkers, SOD and malondialdehyde, and the results revealed that the concentrations of SOD in the serum and the mRNA expression of CUZnSOD in the adipose tissue were increased and the concentrations of malondialdehyde in the serum were decreased by histidine supplementation, indicating that histidine is a potential antioxidant in obese individuals.

All these findings indicate that histidine supplementation could attenuate inflammation and oxidative stress in obese women. The results of the present study also provide a new insight for understanding the relationship between amino 
acid metabolism disorder and inflammation and/or oxidative stress, which suggests that more attention has to be paid to uncovering amino acid metabolism in obese individuals in a further study. In addition, previous studies have shown that women are more sensitive to dietary histidine and energy intake than men ${ }^{(42,43)}$. Therefore, we chose female rats as the experimental animals in the present study. We established a HH DR group for excluding the effects of the reduced body weight on the alleviation of inflammation. However, artificially controlling food intake cannot completely imitate food intake reduction and body weight loss caused by histidine supplementation-induced appetite suppression. The suppression of appetite might lead to some unknown effects, which might be associated with the regulation of inflammation. Therefore, we cannot completely exclude the possibility that some of the findings might be related to the suppression of appetite, and more studies are required to be conducted in the future.

In summary, the present study provides evidence for the first time that by inhibiting NF-кB and activating PPAR $\gamma$, histidine supplementation protects the adipose tissue from inflammation and oxidative stress induced by HFD feeding, suggesting that histidine is a potential candidate for ameliorating inflammation and oxidative stress in obese individuals

\section{Acknowledgements}

The present study was supported by the National Natural Science Fund of China (81130049 and 81202184), the 12th China Five-Year Scientific and Technical Plan (grant no. 2012BAI02B00), and the Research Fund for Innovation Talents of Science and Technology in Harbin City (2013RFQXJ068).

The authors' contributions are as follows: S. Li, Ying Li and C. S. were responsible for the study concept and design; X. S., R. F. and W. Z. performed the experiment and collected the data; Yanchuan Li and S. Lin carried out the analysis and interpretation of the data; S. Lin and W. Z. performed the statistical analysis; X. S. and R. F. wrote the article; Ying Li and C. S. were responsible for obtaining funds and supervision; S. Li, X. S. and C. S. also contributed to the critical revision of the manuscript for its intellectual content; X. S. and S. Li had primary responsibility for the final content. All authors read and approved the final manuscript.

None of the authors has any conflicts of interest to declare.

\section{References}

1. Greenberg AS \& Obin MS (2006) Obesity and the role of adipose tissue in inflammation and metabolism. Am J Clin Nutr 83, 461S-465S.

2. Trayhurn P \& Wood IS (2004) Adipokines: inflammation and the pleiotropic role of white adipose tissue. Br J Nutr $\mathbf{9 2}$, $347-355$.

3. Mokdad AH, Ford ES, Bowman BA, et al. (2003) Prevalence of obesity, diabetes, and obesity-related health risk factors, 2001. JAMA 289, 76-79.

4. Esposito K \& Giugliano D (2004) The metabolic syndrome and inflammation: association or causation? Nutr Metab Cardiovasc Dis 14, 228-232.
5. Watanabe M, Suliman ME, Qureshi AR, et al. (2008) Consequences of low plasma histidine in chronic kidney disease patients: associations with inflammation, oxidative stress, and mortality. Am J Clin Nutr 87, 1860-1866.

6. Niu YC, Feng RN, Hou Y, et al. (2012) Histidine and arginine are associated with inflammation and oxidative stress in obese women. Br J Nutr 108, 57-61.

7. Feng RN, Niu YC, Sun XW, et al. (2013) Histidine supplementation improves insulin resistance through suppressed inflammation in obese women with the metabolic syndrome: a randomised controlled trial. Diabetologia 56, 985-994.

8. Lee YT, Hsu CC, Lin MH, et al. (2005) Histidine and carnosine delay diabetic deterioration in mice and protect human low density lipoprotein against oxidation and glycation. Eur J Pharmacol 513, 145-150.

9. Son DO, Satsu H \& Shimizu M (2005) Histidine inhibits oxidative stress- and TNF- $\alpha$-induced interleukin- 8 secretion in intestinal epithelial cells. FEBS Lett 579, 4671-4677.

10. Tak PP \& Firestein GS (2001) NF-кB: a key role in inflammatory diseases. J Clin Invest 107, 7-11.

11. Li Q \& Verma IM (2002) NF-kB regulation in the immune system. Nat Rev Immunol 2, 725-734.

12. Yamamoto Y \& Gaynor RB (2001) Therapeutic potential of inhibition of the NF- $\kappa$ B pathway in the treatment of inflammation and cancer. J Clin Invest 107, 135-142.

13. D'Acquisto F, May MJ \& Ghosh S (2002) Inhibition of nuclear factor kappa B (NF-B): an emerging theme in antiinflammatory therapies. Mol Interv 2, 22-35.

14. Finelli C \& Tarantino G (2013) What is the role of adiponectin in obesity related non-alcoholic fatty liver disease? World J Gastroenterol 19, 802-812.

15. Kwon H \& Pessin JE (2013) Adipokines mediate inflammation and insulin resistance. Front Endocrinol (Lausanne) 4, 71 .

16. Ouchi N \& Walsh K (2007) Adiponectin as an anti-inflammatory factor. Clin Chim Acta 380, 24-30.

17. Maeda N, Takahashi M, Funahashi T, et al. (2001) PPAR $\gamma$ ligands increase expression and plasma concentrations of adiponectin, an adipose-derived protein. Diabetes 50, 2094-2099.

18. Monsalve FA, Pyarasani RD, Delgado-Lopez F, et al. (2013) Peroxisome proliferator-activated receptor targets for the treatment of metabolic diseases. Mediators Inflamm 2013, 549627.

19. Reeves PG (1997) Components of the AIN-93 diets as improvements in the AIN-76A diet. J Nutr 127, 838S-841S.

20. Yan SL, Wu ST, Yin MC, et al. (2009) Protective effects from carnosine and histidine on acetaminophen-induced liver injury. J Food Sci 74, H259-H265.

21. Liu WH, Liu TC \& Yin MC (2008) Beneficial effects of histidine and carnosine on ethanol-induced chronic liver injury. Food Chem Toxicol 46, 1503-1509.

22. Li S, Li Y, Ning H, et al. (2013) Calcium supplementation increases circulating cholesterol by reducing its catabolism via GPER and TRPC1-dependent pathway in estrogen deficient women. Int J Cardiol 168, 2548-2560.

23. Dong YM, Li Y, Ning H, et al. (2011) High dietary intake of medium-chain fatty acids during pregnancy in rats prevents later-life obesity in their offspring. J Nutr Biochem 22, 791-797.

24. Mihalik SJ, Michaliszyn SF, de las Heras J, et al. (2012) Metabolomic profiling of fatty acid and amino acid metabolism in youth with obesity and type 2 diabetes: evidence for enhanced mitochondrial oxidation. Diabetes Care 35 , 605-611. 
25. Kasaoka S, Tsuboyama-Kasaoka N, Kawahara Y, et al. (2004) Histidine supplementation suppresses food intake and fat accumulation in rats. Nutrition 20, 991-996.

26. Yoshimatsu H, Chiba S, Tajima D, et al. (2002) Histidine suppresses food intake through its conversion into neuronal histamine. Exp Biol Med (Maywood) 227, 63-68.

27. Gustafson B (2010) Adipose tissue, inflammation and atherosclerosis. J Atheroscler Thromb 17, 332-341.

28. Kern PA, Ranganathan S, Li C, et al. (2001) Adipose tissue tumor necrosis factor and interleukin-6 expression in human obesity and insulin resistance. Am $J$ Physiol Endocrinol Metab 280, E745-E751.

29. Yudkin JS, Stehouwer CD, Emeis JJ, et al. (1999) C-reactive protein in healthy subjects: associations with obesity, insulin resistance, and endothelial dysfunction: a potential role for cytokines originating from adipose tissue? Arterioscler Thromb Vasc Biol 19, 972-978.

30. Kang JH, Goto T, Han IS, et al. (2010) Dietary capsaicin reduces obesity-induced insulin resistance and hepatic steatosis in obese mice fed a high-fat diet. Obesity (Silver Spring) 18, 780-787.

31. Chuang CC \& McIntosh MK (2011) Potential mechanisms by which polyphenol-rich grapes prevent obesity-mediated inflammation and metabolic diseases. Annu Rev Nutr 31. 155-176.

32. Baker RG, Hayden MS \& Ghosh S (2011) NF-кB, inflammation, and metabolic disease. Cell Metab 13, 11-22.

33. Arkan MC, Hevener AL, Greten FR, et al. (2005) IKK- $\beta$ links inflammation to obesity-induced insulin resistance. Nat Med 11, 191-198.

34. Andou A, Hisamatsu T, Okamoto S, et al. (2009) Dietary histidine ameliorates murine colitis by inhibition of proinflammatory cytokine production from macrophages. Gastroenterology 136, 564-574. e562.

35. Yang WS, Lee WJ, Funahashi T, et al. (2001) Weight reduction increases plasma levels of an adipose-derived anti-inflammatory protein, adiponectin. J Clin Endocrinol Metab 86, 3815-3819.

36. Chung SW, Kang BY, Kim SH, et al. (2000) Oxidized low density lipoprotein inhibits interleukin-12 production in lipopolysaccharide-activated mouse macrophages via direct interactions between peroxisome proliferator-activated receptor-gamma and nuclear factor-kappa B. J Biol Chem 275, 32681-32687.

37. Remels AH, Langen RC, Gosker HR, et al. (2009) PPARy inhibits NF-кB-dependent transcriptional activation in skeletal muscle. Am J Physiol Endocrinol Metab 297, E174-E183.

38. Seymour EM, Bennink MR, Watts SW, et al. (2010) Whole grape intake impacts cardiac peroxisome proliferatoractivated receptor and nuclear factor kappaB activity and cytokine expression in rats with diastolic dysfunction. Hypertension 55, 1179-1185.

39. Kulebyakin K, Karpova L, Lakonsteva E, et al. (2012) Carnosine protects neurons against oxidative stress and modulates the time profile of MAPK cascade signaling. Amino Acids 43, 91-96.

40. Babizhayev MA, Seguin MC, Gueyne J, et al. (1994) L-carnosine ( $\beta$-alanyl-L-histidine) and carcinine ( $\beta$-alanylhistamine) act as natural antioxidants with hydroxyl-radical-scavenging and lipid-peroxidase activities. Biochem $J$ 304, Pt 2, 509-516.

41. Lee JW, Miyawaki H, Bobst EV, et al. (1999) Improved functional recovery of ischemic rat hearts due to singlet oxygen scavengers histidine and carnosine. $\mathrm{J} \mathrm{Mol}$ Cell Cardiol 31, 113-121.

42. Kasaoka S, Kawahara Y, Inoue S, et al. (2005) Gender effects in dietary histidine-induced anorexia. Nutrition 21, 855-858.

43. Okubo H \& Sasaki S (2005) Histidine intake may negatively correlate with energy intake in human: a cross-sectional study in Japanese female students aged 18 years. J Nutr Sci Vitaminol (Tokyo) 51, 329-334. 